\title{
A Pseudo Random Postfix OFDM Based Modulator for Multiple Antennæ Systems
}

\author{
Markus Muck*, Alexandre Ribeiro Dias*, Marc de Courville*, Pierre Duhamel ${ }^{\dagger}$ \\ *Motorola Labs, Espace Technologique, 91193 Gif-sur-Yvette, France, Email: Markus.Muck@ motorola.com \\ ${ }^{\dagger}$ CNRS/LSS Supelec, Plateau de moulon, 91192 Gif-sur-Yvette, France
}

\begin{abstract}
This contribution ${ }^{1}$ extends the recently proposed pseudo-random-postfix OFDM (PRP-OFDM) transceiver to the Multiple Transmit Multiple Receive (MTMR) antennæ context. In this paper a new generic MTMR modulator is proposed that grants a low complexity semi-blind identification of all channels between any transmit and receive antenna based on order-one statistics. A new channel tracking strategy is detailed based on a Moving Average (MA) Doppler modeling that preserves the Jakes channel coefficients correlation properties. Moreover two transceiver architectures are presented with an Alamouti based Space-Time (ST) block code: equalization schemes with or without postfix interference cancellation and related modulators. Performance is illustrated by simulations in the context of the 5.2GHz OFDM WLAN IEEE802.11a standard.
\end{abstract}

\section{INTRODUCTION}

OFDM is the chosen modulation scheme of modern highdata rate communication systems (xDSL, DAB, DVB-T, IEEE802.11a/g, IEEE802.15.3a, considered for 4G, etc.). In the context of WLANs, current data rates $(54 \mathrm{Mbps}$ on top of the physical layer) are foreseen to be insufficient for very dense urban deployment, such as for hot spot coverage. This is the motivation for IEEE to propose and specify in the scope of the IEEE802.11n solutions for very high data rates WLANs (targeting at least $100 \mathrm{Mbps}$ on top of the MAC layer) in the $5 \mathrm{GHz}$ band. Another path is to investigate higher frequency bands where more spectrum is available such as the $60 \mathrm{GHz}$ [1]. One way of achieving higher data rates is to provide the system with multiple antennæ both at the transmitter and receiver sides. By doing so, it is possible to increase the quality of the communication link by exploiting the spatial diversity dimension using for instance Space Time Block Codes (STBC) [2], or to increase the spectral efficiency of the system by transmitting simultaneously different streams using Spatial Division Multiplexing (SDM) [3]. Therefore, Multiple Transmit Multiple Receive (MTMR) antennæ systems are strong candidates for next generation WLANs.

All the existing OFDM systems are based on a traditional Cyclic Prefix OFDM (CP-OFDM) modulation scheme. The role of the cyclic prefix is to turn the linear convolution into a set of parallel attenuations in the discrete frequency domain. Recent contributions have proposed alternatives: replacing this time domain redundancy by null samples leads to the so called Zero Padded OFDM (ZP-OFDM) [4], [5]. This solution relying on a larger FFT demodulator, has the merit

\footnotetext{
${ }^{1}$ This work is supported by the European Commission and is a part of the IST BROADWAY PROJECT IST-2001-32686 [1].
}

to guarantee symbol recovery irrespective of channel null locations in absence of noise when the channel is known. Still, channel estimation remains an issue. Another recent proposal is to replace the time domain redundancy by a pseudorandomly weighted deterministic sequence [6] which leads to the so called Pseudo Random Postfix OFDM (PRP-OFDM); the benefits are to i) preserve the ZP-OFDM advantages and ii) allow low complexity channel estimation at the receiver. Note that PRP-OFDM does not impact the achieved useful data rate and spectral efficiency compared to the classical CP-OFDM modulator since the only modification is the postfix content.

This contribution capitalizes on existing single antenna PRP-OFDM [6] and presents in section II a novel extension of the PRP-OFDM transceiver to the MTMR case. The approach taken does neither limit the number of transmit and receive antennæ nor impose a particular Space-Time Code (STC).

For coherent MTMR systems, the estimation and tracking of the Multiple Input Multiple Output (MIMO) channel is essential and becomes challenging in presence of high Doppler (either considering high mobility scenarios or high frequency bands). In the scheme considered, data and postfix vectors are independently encoded by two STCs; the new specific MTMR postfix design proposed in section III enables a semiblind estimation of all the MIMO channel exploiting only the order-one statistics of the received signal. Moreover after presenting the new channel estimator in the static case, a new one is derived for high mobility scenarii based on a new Doppler model. Compared to order 1 Auto Regressive (AR) Doppler models [5], the one proposed here is based on a Moving Average (MA) and better preserves the Jakes channel coefficients correlations properties for high Doppler spreads.

Section IV proposes two transceiver architectures in the case of an Alamouti based STBC in order to illustrate the decoding and channel estimation steps. Two different decoding strategies are discussed. First one is to convert the received blocks back to ZP-OFDM vectors through postfix contribution cancellation and then decode them using architectures proposed in [4], [5]. Second one is to equalize the full received vector exploiting the diagonalisation properties of pseudo-circulant matrices [6].

Section V finally presents simulation results and Section VI draws conclusions.

\section{PRP-OFDM MODULATION AND DEMODULATION}

This section presents both the digital PRP-OFDM MTMR modulator and a discrete baseband channel model. Figure 1 


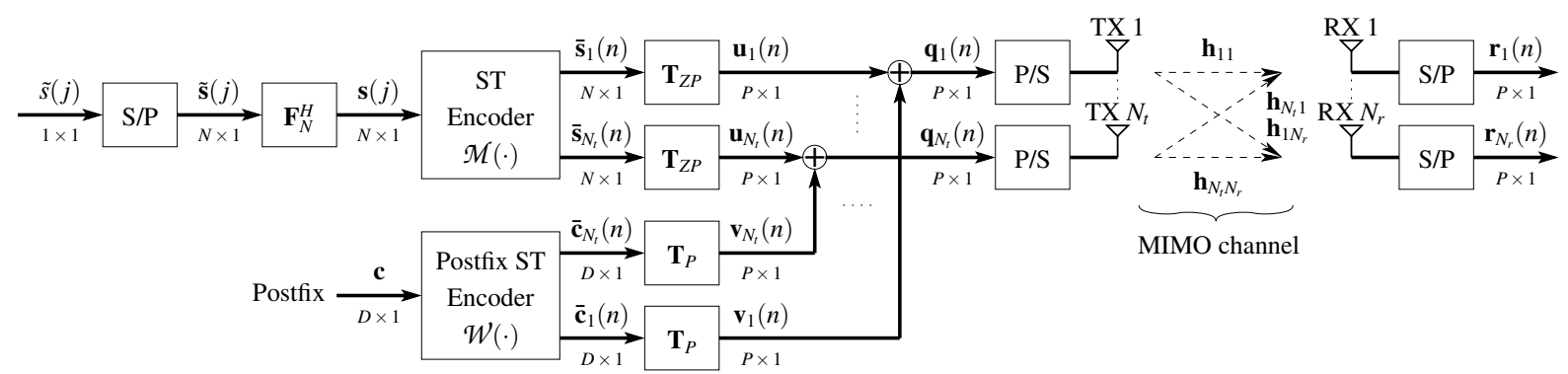

Fig. 1. Discrete model of the MTMR PRP-OFDM modulator.

depicts the baseband discrete-time block equivalent model of a $N$-carrier PRP-OFDM MTMR transceiver with $N_{t}$ transmit and $N_{r}$ receive antennæ. Please note that the proposed scheme is very generic, but for concision sake we will limit the discussion here to Space-Time (ST) block codes. The initial serial stream of constellation symbols $\tilde{s}(j N), \cdots, \tilde{s}(j N+$ $N-1)$ is serial-to-parallel converted; the $j$ th $N \times 1$ input digital vector ${ }^{2} \tilde{\mathbf{s}}(j)$ is then modulated by the IFFT matrix $\mathbf{F}_{N}^{H}$ with $\left[\mathbf{F}_{N}\right]_{k, l}=(1 / \sqrt{N}) W_{N}^{k l}, 0 \leq k<N, 0 \leq l<$ $N$ where $W_{N}=e^{-j 2 \pi / N}$. The resulting $N \times 1$ time domain vector $\mathbf{s}(j)$ is processed by any suitable $\mathrm{ST}$ encoder $\mathcal{M}$ creating the outputs $\overline{\mathbf{S}}(i)=\mathcal{M}\left(\mathbf{s}\left(i N_{t}\right), \cdots, \mathbf{s}\left(i N_{t}+N_{t}-1\right)\right)=$ $\left\{\overline{\mathbf{s}}_{l}(i M+k), 1 \leq l \leq N_{t}, 0 \leq k<M\right\}$ with $i$ being the block number and $n=i M+k$ indexing the outputs in Figure 1. Note that in the context of STBCs, $M$ can differ from $N_{t}$. For example STBCs given in [7] lead to rectangular $\overline{\mathbf{S}}(i)$, i.e. $M>N_{t}$. In the sequel, the $\overline{\mathbf{s}}_{l}(i M+k)$ are linearly precoded by a ZP-OFDM precoding matrix $\mathbf{T}_{Z P}$

$$
\mathbf{T}_{Z P}=\left[\frac{\mathbf{I}_{N}}{\mathbf{0}_{D, N}}\right]_{P \times N}
$$

with $\mathbf{u}_{l}(n)=\mathbf{T}_{Z P} \overline{\mathbf{s}}_{l}(n), 1 \leq l \leq N_{t}$. A pseudo random postfix, chosen with respect to the design criteria given by [6], is appended afterwards. In the MTMR case, the deterministic $D \times 1$ postfix vector c is treated by a specific ST encoder $\mathcal{W}$ which outputs the $D \times 1$ vectors $\overline{\mathbf{c}}_{l}(n), 1 \leq l \leq N_{t}$. As it will be shown later $\mathcal{W}$ is there for ensuring identification of the complete MIMO channel. In order to avoid unpleasant spectrum properties, the postfix vector is weighted by a scalar pseudo-random sequence (cf. [6]). The postfix vectors $\overline{\mathbf{c}}_{l}(n)$ are then linearly precoded by the matrix $\mathbf{T}_{P}$

$$
\mathbf{T}_{P}=\left[\frac{\mathbf{0}_{N, D}}{\mathbf{I}_{D}}\right]_{P \times D}
$$

and the resulting $\mathbf{v}_{l}(n)$ are finally added to the data symbols $\mathbf{u}_{l}(n): \mathbf{q}_{l}(n)=\mathbf{u}_{l}(n)+\mathbf{v}_{l}(n), 1 \leq l \leq N_{t}$. Let $\mathbf{H}_{l m}$ be a $P \times P$ circulant matrix whose first row is given by $\left[h_{l m}(0), 0 \rightarrow\right.$ $\left.0, h_{l m}(L-1), \cdots, h_{l m}(1)\right]$, where $\mathbf{h}_{l m}=\left[h_{l m}(0), \cdots, h_{l m}(L-\right.$ $1), 0 \rightarrow 0]^{T}$ is the $P \times 1$ channel impulse response between the $l$ th transmit and the $m$ th receive antenna; $D$ is chosen

\footnotetext{
${ }^{2}$ Lower (upper) boldface symbols will be used for column vectors (matrices) sometimes with subscripts $N$ or $P$ emphasizing their sizes (for square matrices only); tilde will denote frequency domain quantities; argument $i$ will be used to index blocks of symbols; ${ }^{H}\left({ }^{T}\right)$ will denote Hermitian (transpose); ${ }^{\star}$ will denote complex conjugate.
}

such that $D \geq L-1$. Define $\mathbf{H}_{l m}^{\mathrm{ISI}}$ as the lower triangular part of $\mathbf{H}_{l m}$ including the main diagonal which represents the Intra-Symbol-Interference (ISI); $\mathbf{H}_{l m}^{\mathrm{BI}}$ shall contain the upper triangular part of $\mathbf{H}_{l m}$ representing the Inter-Block-Interference (IBI), such that $\mathbf{H}_{l m}=\mathbf{H}_{l m}^{\mathrm{ISI}}+\mathbf{H}_{l m}^{\mathrm{IBI}}$. Therefore, the received vector on the $m$ th antenna, $1 \leq m \leq N_{r}$ is given by [6]:

$$
\mathbf{r}_{m}(n)=\sum_{l=1}^{N_{t}}\left[\mathbf{H}_{l m}^{\mathrm{ISI}} \mathbf{q}_{l}(n)+\mathbf{H}_{l m}^{\mathrm{IBI}} \mathbf{q}_{l}(n-1)\right]+\mathbf{n}_{m}(n)
$$

where $\mathbf{n}_{m}(n)$ is an zero-mean additive white i.i.d. gaussian noise term.

A choice of the pseudo random postfix ST encoder $\mathcal{W}$ is discussed in the next section in order to allow a simple identification of all channels $\mathbf{h}_{l m}, 1 \leq l \leq N_{t}, 1 \leq m \leq N_{r}$.

\section{ORDER-ONE MIMO CHANNEL ESTIMATION}

The goal of this section is to extend the order-one channel estimation technique presented in [6] to the MTMR case. First, a novel channel estimation algorithm is detailed, assuming the channel to be static. Then, a novel Doppler model is introduced for the mobility case and the corresponding optimum channel estimator in the Minimum Mean Square Error (MMSE) sense.

First let express the received vector $\mathbf{r}_{m}(n)$ in an exploitable form for channel estimation. For that purpose let $\mathbf{H}_{l m}^{D}$ be the $D \times D$ circulant matrix of first row $\left[h_{l m}(0), 0 \rightarrow 0, h_{l m}(L-\right.$ 1), $\left.\cdots, h_{l m}(1)\right]$. We define $\mathbf{H}_{l m}^{\mathrm{ISI}, D}$ and $\mathbf{H}_{l m}^{\mathrm{IB}, D}$ as previously such that $\mathbf{H}_{l m}^{D}=\mathbf{H}_{l m}^{\mathrm{ISI}, D}+\mathbf{H}_{l m}^{\mathrm{IBI}, D}$. The signal $\mathbf{r}_{m}(n)$, received during the $n$th OFDM symbol on the $m$ th antenna, $1 \leq m \leq N_{r}$, is equal to:

$$
\mathbf{r}_{m}(n)=\sum_{l=1}^{N_{t}}\left[\begin{array}{c}
\mathbf{H}_{l m}^{\mathrm{IS} l, D} \overline{\mathbf{s}}_{l, 0}(n)+\mathbf{H}_{l m}^{\mathrm{IBl}, D} \overline{\mathbf{c}}_{l}(n-1) \\
\vdots \\
\mathbf{H}_{l m}^{\mathrm{IBl}, D} \overline{\mathbf{s}}_{l, 1}(n)+\mathbf{H}_{l m}^{\mathrm{ISI}, D} \overline{\mathbf{c}}_{l}(n)
\end{array}\right]+\left[\begin{array}{c}
\mathbf{n}_{m, 0}(n) \\
\vdots \\
\mathbf{n}_{m, 1}(n)
\end{array}\right]
$$

where $\overline{\mathbf{s}}_{l, 0}(n), \overline{\mathbf{s}}_{l, 1}(n), \mathbf{n}_{m, 0}(n), \mathbf{n}_{m, 1}(n)$ are respectively the first $D$ and last $D$ samples of $\overline{\mathbf{s}}_{l}(n)$ and $\mathbf{n}_{m}(n)$.

Equation (1) tells that a superimposition of the various postfixes convolved by the corresponding channels is interfering with the useful data. An easy independant retrieval of each of the channels based on the sole observation of the postfixes contributions would be possible through isolation of each postfix convolved by its related channel. As detailed below, a way to achieve that condition is to use a weighting ST block coding scheme $\mathcal{W}$ of the postfix $\mathbf{c}$ using the following 
postfixes generation process :

$$
\begin{aligned}
& {\left[\begin{array}{ccc}
\overline{\mathbf{c}}_{1}(i M) & \cdots & \overline{\mathbf{c}}_{1}(i M+M-1) \\
\vdots & \ddots & \vdots \\
\overline{\mathbf{c}}_{N_{t}}(i M) & \cdots & \overline{\mathbf{c}}_{N_{t}}(i M+M-1)
\end{array}\right]=} \\
& \underbrace{\left[\begin{array}{ccc}
w_{1}(0) \alpha(i M) & \cdots & w_{1}(M-1) \alpha(i M+M-1) \\
\vdots & \ddots & \vdots \\
w_{N_{t}}(0) \alpha(i M) & \cdots & w_{N_{t}}(M-1) \alpha(i M+M-1)
\end{array}\right]}_{\mathcal{W}} \otimes \mathbf{c}
\end{aligned}
$$

where $\otimes$ is the Kronecker product and $\mathbf{c}, \alpha(n)$ are respectively the deterministic postfix and the pseudo-random weighting factors introduced in [6]. The pseudo-random weighting factors $\alpha(n)$ are used to convert the deterministic postfix $\mathbf{c}$ into a pseudo-random one. Note that a new set of deterministic weighting factors is introduced, and gathered in the $M \times N_{t}$ matrix $\mathbf{W}$, with $[\mathbf{W}]_{k, l-1}=w_{l}(k), 0 \leq k<M, 1 \leq l \leq N_{t}$. W is there to remove the interference between all transmitted postfixes and thus needs to be invertible: $\mathbf{W}$ is of full column rank $\left(\operatorname{rank}(\mathbf{W})=N_{t}\right)$. In the following we choose $\mathbf{W}$ orthogonal, such that $\mathbf{W}^{H} \mathbf{W}=N_{t} \times \mathbf{I}_{N_{t}}$.

\section{A. Static context}

Let focus now on a static channel and detail an order-one channel estimator. For that purpose, denote respectively by $\mathbf{r}_{m, 0}(n)$ and $\mathbf{r}_{m, 1}(n)$ the first and last $D$ samples of $\mathbf{r}_{m}(n)$. By setting $n=i M+k$ and assuming the transmitted time domain signal $\overline{\mathbf{s}}_{l}(n)$ to be zero mean for all $l$, we use (2) and (1) to compute for each $k, 0 \leq k<M$, the following $D \times 1$ vector:

$$
\mathbf{d}_{m}^{k}(i)=\frac{\mathbf{r}_{m, 1}(i M+k)+\mathbf{r}_{m, 0}(i M+k+1)}{\alpha(i M+k)}
$$

Define $\mathbf{d}_{m}^{k}=\mathrm{E}\left[\mathbf{d}_{m}^{k}(i)\right]$ as the expectation of $\mathbf{d}_{m}^{k}(i)$. Thanks to the deterministic nature of the postfixes, it can be verified from (1) that:

$$
\mathbf{d}_{m}^{k}=\sum_{l=1}^{N_{t}}\left[\mathbf{H}_{l m}^{\mathrm{ISI}, D}+\mathbf{H}_{l m}^{\mathrm{IBI}, D}\right] w_{l}(k) \mathbf{c}=\sum_{l=1}^{N_{t}} \mathbf{H}_{l m}^{D} w_{l}(k) \mathbf{c}
$$

Thus the $M \cdot D \times 1$ vector $\mathbf{d}_{m}=\left[\mathbf{d}_{m}^{0 T}, \cdots,\left(\mathbf{d}_{m}^{M-1}\right)^{T}\right]^{T}$ can be expressed for each receive antenna as:

$$
\mathbf{d}_{m}=\sum_{l=1}^{N_{t}}\left[\begin{array}{c}
\mathbf{H}_{l m}^{D} w_{l}(0) \mathbf{c} \\
\vdots \\
\mathbf{H}_{l m}^{D} w_{l}(M-1) \mathbf{c}
\end{array}\right]=\left(\mathbf{W} \otimes \mathbf{I}_{D}\right) \cdot\left[\begin{array}{c}
\mathbf{H}_{1 m}^{D} \mathbf{c} \\
\vdots \\
\mathbf{H}_{N_{t} m}^{D} \mathbf{c}
\end{array}\right]
$$

Since $\mathbf{W}$ is chosen orthogonal, multiplying each $\mathbf{d}_{m}, 1 \leq m \leq$ $N_{r}$ by $\left(\mathbf{W} \otimes \mathbf{I}_{D}\right)^{H}$ removes completely the interference between channel contributions $\mathbf{H}_{l m}^{D}, 1 \leq l \leq N_{t}$.

Once the interference between channel contributions is removed (each vector $\mathbf{H}_{l m}^{D} \mathbf{c}, 1 \leq l \leq N_{t}$ in (4) is isolated), estimation algorithms of the single-antenna case [6] apply:

$$
\mathbf{H}_{l m}^{D} \mathbf{c}=\mathbf{C}_{D} \mathbf{h}_{l m}^{D}=\mathbf{F}_{D}^{H} \tilde{\mathbf{C}}_{D} \mathbf{F}_{D} \mathbf{h}_{l m}^{D}
$$

where $\mathbf{C}_{D}$ is a $D \times D$ circulant matrix with the first row $[c(0), c(D-1), \cdots, c(1)], \tilde{\mathbf{C}}_{D}=\operatorname{diag}\left\{\mathbf{F}_{D} \mathbf{c}\right\}$, and $\mathbf{h}_{l m}^{D}$ represents the $D$ first coefficients of $\mathbf{h}_{l m}$. Hence, the estimate $\hat{\mathbf{h}}_{l m}^{D}$ of the time domain channel impulse response $\mathbf{h}_{l m}^{D}$ is obtained by multiplying $\mathbf{H}_{l m}^{D} \mathbf{c}$ by $\mathbf{F}_{D}^{H} \tilde{\mathbf{C}}_{D}^{-1} \mathbf{F}_{D}, 1 \leq l \leq N_{t}, 1 \leq m \leq N_{r}$. Note that $\tilde{\mathbf{C}}_{D}^{-1}$ is a diagonal matrix that is a-priori known to both the transmitter and receiver and can thus be precalculated. Subsequently, $\hat{\mathbf{h}}_{l m}^{D}$ is usually transformed to the $P \times 1$ frequency domain vector $\hat{\tilde{\mathbf{h}}}_{l m}=\mathbf{F}_{P}\left[\mathbf{I}_{D}^{T}, \mathbf{0}_{N, D}^{T}\right]^{T} \hat{\mathbf{h}}_{l m}^{D}$.

\section{B. Doppler Context}

Let extend the above channel estimator to mobile environments. For that purpose, a Doppler model is introduced as a modification of the standard order-one AR model applied to each Channel Impulse Response (CIR) between transmit antenna $l$ and receive antenna $m$ separately [5]: $\mathbf{h}_{l m}^{D}(n)=$ $J_{0}\left(2 \pi f_{D} \Delta T\right) \mathbf{h}_{l m}^{D}(n-1)+\breve{\mathbf{h}}_{l m}^{D}(n)$ where $J_{0}(\cdot)$ is the 0th order Bessel function, $f_{D}$ is the Doppler frequency, $\Delta T$ is the MTMR PRP-OFDM block duration and $\breve{\mathbf{h}}_{l m}^{D}(n)$ is zeromean complex gaussian of constant variance. The idea is to guarantee to have same CIR correlations than the ones provided by the well known Jakes model [8] even in the presence of large Doppler frequencies. This is achieved by forcing the correlation $\mathrm{E}\left[\left(\mathbf{h}_{l m}^{D}(n)\right)^{H} \mathbf{h}_{l m}^{D}(k)\right]=J_{0}\left(2 \pi f_{D}(k-\right.$ $n) \Delta T), \forall k>n$. This way the approximation $J_{0}\left(2 \pi f_{D}(k-\right.$ $n) \Delta T) \approx\left(J_{0}\left(2 \pi f_{D} \Delta T\right)\right)^{k-n}$, inherent to the order-one AR model, is avoided. This modification leads to the following MA model:

$$
\mathbf{h}_{l m}^{D}(n)=\sum_{k=0}^{n} J_{0}\left(2 \pi f_{D} k \Delta T\right) \breve{\mathbf{h}}_{l m}^{D}(n-k) .
$$

As for the order-one AR model [5], the so-called processnoise vectors $\breve{\mathbf{h}}_{l m}^{D}(n)$ are introduced assuming $\mathrm{E}\left[\breve{\mathbf{h}}_{l m}^{D}(k) \breve{\mathbf{h}}_{l m}^{D}(n)^{H}\right]$ $=\mathbf{0}$ for $n \neq k$ and $\breve{\mathbf{h}}_{l m}^{D}(0)=\mathbf{h}_{l m}^{D}(0)$ being the CIR to be estimated. Let assume that CIR $\mathbf{h}_{l m}^{D}$ is estimated based on $Z$ noisy observations $\overline{\mathbf{d}}_{l m}(i)=\left\{\breve{\mathbf{d}}_{l m}(k), i \leq k<i+Z\right\}$ preceding the latest symbol to be decoded. $\breve{\mathbf{d}}_{l m}(i)=\left[\left(\mathbf{W} \otimes \mathbf{I}_{D}\right)^{H} \mathbf{d}_{m}(i)\right]_{k},(l-$ 1) $D \leq k<l D$ results from the convolution of the $i$ th block postfix by channel $\mathbf{h}_{l m}^{D}$ corrupted both by thermic noise and the OFDM data symbols, the latter one is assumed zero-mean and independant of same variance as the postfix samples. $\mathbf{d}_{m}(i)=\left[\mathbf{d}_{m}^{0}(i)^{T}, \cdots, \mathbf{d}_{m}^{M-1}(i)^{T}\right]^{T}$ contains the received symbols after equalization of the pseudo random weighting factor of the postfixes (3). Thus, $\overline{\mathbf{d}}_{l m}(i)$ can be expressed as follows:

$$
\overline{\mathbf{d}}_{l m}(i)=\left(\mathbf{J} \otimes \mathbf{C}_{D}\right) \cdot\left[\breve{\mathbf{h}}_{l m}^{D}(0)^{T}, \cdots, \breve{\mathbf{h}}_{l m}^{D}(Z-1)^{T}\right]^{T}+\mathbf{n},
$$

where

$$
\mathbf{J}=\left[\begin{array}{cccc}
1 & 0 & \cdots & 0 \\
J_{0}\left(2 \pi f_{D} \Delta T\right) & 1 & \cdots & 0 \\
\vdots & \vdots & \ddots & \vdots \\
J_{0}\left(2 \pi f_{D}(Z-1) \Delta T\right) & J_{0}\left(2 \pi f_{D}(Z-2) \Delta T\right) & \cdots & 1
\end{array}\right]
$$

where $\mathbf{n}$ gathers the thermic noise and the interference from the OFDM data symbols. Here, only PRP-OFDM symbols preceding the latest symbol are considered for estimation. In order to guarantee the unit variance of each channel realisation, the norm of $\breve{\mathbf{h}}_{l m}^{D}(n), n=0, \ldots, Z-1$ is chosen such that:

$$
\sum_{n=0}^{Z-1}[\mathbf{J}]_{k, n}^{2} \mathrm{E}\left[\left(\breve{\mathbf{h}}_{l m}^{D}(n)\right)^{H} \breve{\mathbf{h}}_{l m}^{D}(n)\right]=1, k=0, \ldots, Z-1
$$


It can thus be verified that the optimum estimator of $\mathbf{h}_{l m}^{D}(0)=$ $\breve{\mathbf{h}}_{l m}^{D}(0)$ in the MMSE sense is given from (5) by:

$$
\hat{\mathbf{h}}_{l m}^{D}(i)=\mathbf{M}\left[\left(\mathbf{J} \otimes \mathbf{C}_{D}\right) \mathbf{D}_{\mathbf{R}_{\mathbf{h}}}\left(\mathbf{J} \otimes \mathbf{C}_{D}\right)^{H}+\mathbf{R}_{\mathbf{n}}\right]^{-1} \overline{\mathbf{d}}_{m}(i)
$$

with:

$$
\begin{aligned}
\mathbf{D}_{\mathbf{R}_{\mathbf{h}}} & =\text { blockdiag }\left\{\mathbf{R}_{\breve{\mathbf{h}}_{l m}^{D}(0)}, \cdots, \mathbf{R}_{\breve{h}_{l m}^{D}(Z-1)}\right\}, \\
\mathbf{M} & =\left[1, \cdots, J_{0}\left(2 \pi f_{D}(Z-1) \Delta T\right)\right] \otimes\left(\mathbf{R}_{\breve{h}_{l m}^{D}(0)} \mathbf{C}_{D}^{H}\right) .
\end{aligned}
$$

$\mathbf{R}_{\mathbf{a}}=\mathrm{E}\left[\mathbf{a a}^{H}\right]$ is the auto-correlation matrix of the vector $\mathbf{a}$.

Since in practice the channel power profile is usually not known, one can make the assumption that $\mathbf{R}_{\breve{h}_{l m}^{D}(n)} \approx g_{n} \mathbf{I}_{D}$ for all $n$. The real gain $g_{n}$ is introduced for respecting the power constraints of (6).

In order to improve the performances of the channel estimation, it is possible not only to use symbols preceding the latest OFDM symbol for channel estimation, but also following symbols; the drawback is an increase in system latency. Here, in (5) the following observations are considered: $\left[\breve{\mathbf{h}}_{l m}^{D}(-(Z-1) / 2)^{T}, \cdots, \breve{\mathbf{h}}_{l m}^{D}(0)^{T}, \cdots, \breve{\mathbf{h}}_{l m}^{D}((Z-1) / 2)^{T}\right]^{T}$ and the matrices $\mathbf{J}, \mathbf{M}$ and $\mathbf{D}_{\mathbf{R}_{\mathbf{h}}}$ must be adapted adequately.

\section{EXAMPLES OF TRANSCEIVER DESIGNS}

This section presents two Alamouti [2] based modulators that are adapted to the use of pseudo-random postfixes at the transmitter and also to the equalizer structures detailed in this section. The two equalizers proposed are based on the ones already derived for the Single Transmit Single Receive (STSR) case in [6]: one is based on the transformation of the received PRP-OFDM vector to the ZP-OFDM case, the other one on the equalization of the full received block.

The system of interest is chosen to have $N_{t}=2$ transmit and $N_{r}=1$ receive antennæ. The ST encoder operates over $N_{t} \times M$ vectors with $M=N_{t}=2$. Since $N_{r}=1$, the subscript $1 \leq m \leq N_{r}$ is not used in the sequel. A perfect knowledge of the channels $\mathbf{h}_{l}, 1 \leq l \leq N_{t}$ is assumed.

\section{A. ZP-OFDM based decoding approach}

Let apply at the transmit the $2 \times 1 \mathrm{ST}$ encoder $\mathcal{M}$ proposed by [9] in the ZP single-carrier context, which takes two consecutive OFDM symbols $\mathbf{s}(2 i)$ and $\mathbf{s}(2 i+1)$ to form the following coded matrix:

$$
\left[\begin{array}{ll}
\overline{\mathbf{s}}_{1}(2 i) & \overline{\mathbf{s}}_{1}(2 i+1) \\
\overline{\mathbf{s}}_{2}(2 i) & \overline{\mathbf{s}}_{2}(2 i+1)
\end{array}\right]=\left[\begin{array}{cc}
\mathbf{s}(2 i) & -\mathbf{P}_{N}^{0} \mathbf{s}^{\star}(2 i+1) \\
\mathbf{s}(2 i+1) & \mathbf{P}_{N}^{0} \mathbf{s}^{\star}(2 i)
\end{array}\right]
$$

where the permutation matrices $\mathbf{P}_{J}^{n}$ are such that, for a $J \times$ 1 vector $\mathbf{a}=[a(0), \cdots, a(J-1)]^{T},\left\{\mathbf{P}_{J}^{n} \mathbf{a}\right\}_{p}=a((J-1-p+$ $n) \bmod J)$, with $0 \leq p \leq J-1$. Note that (7) reduces to the Alamouti ST block code [2] if $N=1$.

Since the channel is known, as for the single antenna case in [6], it is always possible to retrieve the MTMR ZP-OFDM signals from (1) by substracting from the received signal the known PRP contribution:

$$
\mathbf{r}^{Z P}(n)=\mathbf{r}(n)-\sum_{l=1}^{2}\left[\mathbf{H}_{l}^{\mathrm{IBI}} \mathbf{v}_{l}(n-1)+\mathbf{H}_{l}^{\mathrm{ISI}} \mathbf{v}_{l}(n)\right]
$$

which leads to $\mathbf{r}^{Z P}(n)=\sum_{l=1}^{2} \mathbf{H}_{l} \mathbf{T}_{Z P} \overline{\mathbf{s}}_{l}(n)+\mathbf{n}(n)$. Note that i) no constraint has to be set on $\mathbf{W}$ for the symbol recovery, ii) the PRP interference cancellation procedure proposed is generic and can be applied to any ST encoder $\mathcal{M}$.

Now the same detection algorithm as in [9] can be applied to the signal in (8). Noticing that $\mathbf{P}_{P}^{N} \mathbf{T}_{Z P}=\mathbf{T}_{Z P} \mathbf{P}_{N}^{0}$, we denote by $\tilde{\mathbf{D}}_{1}=\operatorname{diag}\left\{\tilde{\mathbf{h}}_{1}\right\}, \tilde{\mathbf{D}}_{2}=\operatorname{diag}\left\{\tilde{\mathbf{h}}_{2}\right\}, \tilde{\mathbf{n}}(2 i)=\mathbf{F}_{P} \mathbf{n}(2 i)$ and $\tilde{\mathbf{n}}(2 i+$ 1) $=\mathbf{F}_{P} \mathbf{P}_{P}^{N} \mathbf{n}^{\star}(2 i+1)$; if we switch to the frequency domain by computing $\tilde{\mathbf{y}}(2 i)=\mathbf{F}_{P} \mathbf{r}^{Z P}(2 i)$ and $\tilde{\mathbf{y}}(2 i+1)=\mathbf{F}_{P}\left(\mathbf{P}_{P}^{N} \mathbf{r}^{Z P}(2 i+\right.$ 1) $)^{\star}$, exploiting the fact that $\mathbf{H}_{l}=\mathbf{F}_{P}^{H} \tilde{\mathbf{D}}_{l} \mathbf{F}_{P}, 1 \leq l \leq 2$, we can write as in [9]:

$$
\left[\begin{array}{c}
\tilde{\mathbf{y}}(2 i) \\
\tilde{\mathbf{y}}(2 i+1)
\end{array}\right]=\underbrace{\left[\begin{array}{cc}
\tilde{\mathbf{D}}_{1} & \tilde{\mathbf{D}}_{2} \\
\tilde{\mathbf{D}}_{2}^{\star} & -\tilde{\mathbf{D}}_{1}^{\star}
\end{array}\right]}_{=\tilde{\mathbf{D}}}\left[\begin{array}{c}
\mathbf{F}_{P} \mathbf{T}_{Z P} \mathbf{s}(2 i) \\
\mathbf{F}_{P} \mathbf{T}_{Z P} \mathbf{s}(2 i+1)
\end{array}\right]+\left[\begin{array}{c}
\tilde{\mathbf{n}}(2 i) \\
\tilde{\mathbf{n}}(2 i+1)
\end{array}\right]
$$

where $\tilde{\mathbf{D}}$ is an orthogonal channel matrix. Thus multiplying $\left[\tilde{\mathbf{y}}(2 i)^{T}, \tilde{\mathbf{y}}(2 i+1)^{T}\right]^{T}$ by $\tilde{\mathbf{D}}^{H}$ achieves the separation of the transmitted signals $\mathbf{s}(2 i)$ and $\mathbf{s}(2 i+1)$, and it can be shown [9] that full transmit diversity is achieved. Note that the separation of signals allows to use the same equalization schemes as in the single-antenna case [6].

\section{B. Decoding based on diagonalization of pseudo-circulant channel matrices}

The ST data encoder $\mathcal{M}$ considered here is based on a modification version of the Alamouti scheme [2] and outputs blocks of $N_{t} \times M$ vectors with $N_{t}=M=2$. The modification proposed are required for enabling the equalization structure that is detailed below. $\mathcal{M}$ and $\mathcal{W}$ are specified such that they generate the following matrix $\mathbf{Q}(i)=\left\{\mathbf{q}_{l}(2 i+k), 1 \leq l \leq\right.$ $2,0 \leq k<2\}$ at the antennæ outputs:

$$
\mathbf{Q}(i)=\left[\begin{array}{cc}
{\left[\begin{array}{c}
\mathbf{s}(2 i) \\
\alpha(2 i) \mathbf{c}
\end{array}\right]} & -\mathbf{P}_{P}^{0} \mathbf{Q}_{\beta(i)}\left[\begin{array}{c}
\mathbf{s}^{\star}(2 i+1) \\
\beta^{\star}(i) \alpha^{\star}(2 i+1) \mathbf{c}^{\star}
\end{array}\right] \\
{\left[\begin{array}{c}
\mathbf{s}(2 i+1) \\
\alpha(2 i) \mathbf{c}
\end{array}\right]} & \mathbf{P}_{P}^{0} \mathbf{Q}_{\beta(i)}\left[\begin{array}{c}
\mathbf{s}^{\star}(2 i) \\
\beta^{\star}(i) \alpha^{\star}(2 i+1) \mathbf{c}^{\star}
\end{array}\right]
\end{array}\right]
$$

$\mathbf{P}_{P}^{0}$ being a permutation matrix defined as previously (inversing the order of the vector elements), $\alpha(i) \in \mathbb{C}$ with $|\alpha(i)|=1$ being pseudo-random complex weighting factors as defined in [6] with $\alpha(2 i+1)=\beta(i) \alpha(2 i)$ and $\beta(i)=\alpha^{\star}(2 i-1) / \alpha(2 i)$. $\mathbf{Q}_{\beta(i)}$ is defined as:

$$
\mathbf{Q}_{\beta(\mathbf{i})}=\left[\begin{array}{cc}
\mathbf{0}_{D \times N} & \beta(i) \cdot \mathbf{I}_{D} \\
\mathbf{I}_{N} & \mathbf{0}_{N \times D}
\end{array}\right] .
$$

The $D \times 1$ postfix vector $\mathbf{c}$ is chosen such that it has hermitian symmetry, i.e. backwards $\left(\mathbf{c}^{\star}\right)=\mathbf{c}$. Similar to [6], the channels are represented by $P \times P$ pseudo-circulant channel matrices $\mathbf{H}_{l}^{\beta(i)}, 1 \leq l \leq 2$. These are identical to standard circulant convolution matrices with the upper triangular part multiplied by the scalar factor $\beta(i)$, i.e. $\mathbf{H}_{l}^{\beta(i)}=\mathbf{H}_{l}^{\mathrm{ISI}}+\beta(i) \mathbf{H}_{l}^{\mathrm{BII}}$. With $\mathbf{R}(i)=\left[\begin{array}{ll}\mathbf{r}^{T}(2 i) & \mathbf{r}^{T}(2 i+1)\end{array}\right]^{T}$ and the noise matrix $\mathbf{N}(i)=\left[\mathbf{n}^{T}(2 i) \mathbf{n}^{T}(2 i+1)\right]^{T}$, the received signals over $M=2$ symbol times are given as follows:

$$
\mathbf{R}(i)=\left[\begin{array}{c}
\sum_{l=1}^{2}\left[\mathbf{H}_{l}^{\mathrm{BI}} \mathbf{q}_{l}(2 i-1)+\mathbf{H}_{l}^{\mathrm{ISI}} \mathbf{q}_{l}(2 i)\right] \\
\sum_{l=1}^{2}\left[\mathbf{H}_{l}^{\mathrm{IBl}} \mathbf{q}_{l}(2 i)+\mathbf{H}_{l}^{\mathrm{ISI}} \mathbf{q}_{l}(2 i+1)\right]
\end{array}\right]+\mathbf{N}(i)
$$


Exploiting the fact that $\left(\mathbf{P}_{P}^{0} \mathbf{H}_{l}^{\beta(i)} \mathbf{P}_{P}^{0}\right)^{\star}=\left(\mathbf{H}_{l}^{\beta(i)}\right)^{H}$ and $\mathbf{Q}_{\beta(i)} \mathbf{H}_{l}^{\beta(i)}=\mathbf{H}_{l}^{\beta(i)} \mathbf{Q}_{\beta(i)}$, since both are diagonal on the same basis, we compute:

$$
\begin{aligned}
& \hat{\mathbf{R}}(i)=\left[\begin{array}{c}
\mathbf{r}(2 i)+2 \mathbf{c}_{1}^{\beta(i)}(i) \\
\mathbf{Q}_{\beta(i)}^{H}\left(\mathbf{P}_{P}^{0}\left(\mathbf{r}(2 i+1)+\mathbf{c}_{2}^{\beta(i)}(i)\right)\right)^{\star}
\end{array}\right] \\
& =\underbrace{\left[\begin{array}{cc}
\mathbf{H}_{1}^{\beta(i)} & \mathbf{H}_{2}^{\beta(i)} \\
\left(\mathbf{H}_{2}^{\beta(i)}\right)^{H} & -\left(\mathbf{H}_{1}^{\beta(i)}\right)^{H}
\end{array}\right]}_{=\mathbf{W}_{H}(i)}\left[\begin{array}{c}
{\left[\begin{array}{l}
\mathbf{s}(2 i) \\
\alpha(2 i) \mathbf{c}
\end{array}\right]} \\
{\left[\begin{array}{l}
\mathbf{s}(2 i+1) \\
\alpha(2 i) \mathbf{c}
\end{array}\right]}
\end{array}\right], \\
& \mathbf{c}_{1}^{\beta(i)}(i)=\alpha(2 i) \beta(i) \mathbf{H}_{1}^{\mathrm{IB}} \mathbf{T}_{P} \mathbf{c}, \\
& \mathbf{c}_{2}^{\beta(i)}(i)=-2\left[\operatorname{Re}\{\alpha(2 i)\} \mathbf{H}_{1}^{\mathrm{BII}}+j \operatorname{Im}\{\alpha(2 i)\} \mathbf{H}_{2}^{\mathrm{IBI}]} \mathbf{T}_{P} \mathbf{c} .\right.
\end{aligned}
$$

Corresponding to Alamouti's derivations [2], the data symbols can be straightforwardly separated by premultiplication of $\hat{\mathbf{R}}(i)$ by the hermitian of the upper channel matrix $\mathbf{W}_{H}(i)$, since $\mathbf{W}_{H}^{H}(i) \mathbf{W}_{H}(i)$ is a block diagonal matrix. The equalization based on pseudo circulant channel matrices is then performed on $\mathbf{W}_{H}^{H}(i) \hat{\mathbf{R}}(i)$, as presented in [6]. The MIMO channel estimation presented in section III is performed on $\mathbf{R}(i)$.

\section{Simulation RESUlts}

Figure 2 presents Bit Error Rate (BER) simulation results that have been obtained with a MTMR system with two transmit and one receive antennæ in the context of the $5.2 \mathrm{GHz}$ IEEE802.11a WLAN standard. All simulations have been performed for QPSK symbols, a code rate of $R=1 / 2$ and uncorrelated BRAN-A channels [10] with mean unit power. The frame length is set to 432 Bytes of uncoded data, each transmitted over 2000 channel realizations. Different mobility conditions have been considered between $0 \mathrm{~m} / \mathrm{s}$ and $30 \mathrm{~m} / \mathrm{s}$. At the receiver side, the channel power delay profile, the Doppler frequency and the noise variance are assumed to be known. The following systems are compared:

- modified IEEE802.11a standard using Alamouti STBC (CP-OFDM), with ZF equalization and MMSE MIMO channel estimation based on preambles;

- MTMR PRP-OFDM modulator with ZP-OFDM decoding as presented in section IV-A, with MMSE equalization, channel estimation based on 41 received Alamouti blocks of 2 PRP-OFDM symbols (from 20 before to 20 after the latest block). No preambles are introduced.

Without mobility and thanks to the knowledge of the channel power delay profile and noise variance in the receiver, CP-OFDM and PRP-OFDM using MIMO channel estimation show approximately the same performance as CP-OFDM with a perfectly known MIMO channel as presented by Fig.2.

In the presence of mobility and without tracking, CP-OFDM experiences an error floor at a BER of $2 \cdot 10^{-4}$ and $10^{-2}$ for a speed of $10 \mathrm{~m} / \mathrm{s}$ and $20 \mathrm{~m} / \mathrm{s}$ respectively. In turn, at $30 \mathrm{~m} / \mathrm{s}$, PRPOFDM shows practically no performance loss compared to the static case up to a BER of $5 \cdot 10^{-4}$. At a BER of $10^{-5}$, the loss is limited to approximately $2 \mathrm{~dB}$. This robustness is obtained thanks to the PRP-OFDM modulation, for which the MIMO channel tracking method proposed in section III-B applies.

\section{CONCLUSION}

A new OFDM modulation scheme based on pseudo random postfix insertion (referred to as PRP-OFDM) has been presented for multiple antenna systems. It has been shown that the postfix can be, similar to the data stream, encoded based on a suitable STC. This allows a semi-blind identification of the MIMO channel in the receiver requiring a very low arithmetical complexity. The simulation results given for an Alamouti based MTMR system with two transmit and one receive antennæ show that the proposed CIR estimation techniques work very robustly, even in a high mobility scenario (mobility up to $30 \mathrm{~m} / \mathrm{s}$ is considered here). In the same environment, IEEE802.11a shows a considerable performance degradation compared to the static case.

\section{REFERENCES}

[1] M. de Courville, S. Zeisberg, M. Muck, and J. Schoenthier. BroadWay - the way to broadband access at $60 \mathrm{GHz}$. In International Conference on Telecommunication, Beijing, China, June 2002.

[2] S.M. Alamouti. A Simple Transmit Diversity Technique for Wireless Communications. IEEE Journal on Selected Areas in Communications, 16(8):1451-1458, October 1998.

[3] G.J. Foschini. Layered space-time architecture for wireless communication in a fading environment when using multi-element antennas. Bell Labs Tech. J., pages 41-59, Autumn 1996.

[4] B. Muquet, Z. Wang, G. B. Giannakis, M. de Courville, and P. Duhamel. Cyclic Prefixing or Zero Padding for Wireless Multicarrier Transmissions ? IEEE Trans. on Communications, December 2002.

[5] Shengli Zhou, Xiaoli Ma, and Georgios B. Giannakis. Space-Time Coding and Kalman Filtering for Time-Selective Fading Channels. IEEE Transactions on Communications, Volume 50:183 - 186, February 2002.

[6] M. Muck, M. de Courville, M. Debbah, and P. Duhamel. A Pseudo Random Postfix OFDM modulator and inherent channel estimation techniques. In GLOBECOM conference records, San Francisco, USA, December 2003.

[7] V. Tarokh, H. Jafarkhani, and A.R. Calderbank. Space-Time Block Codes from Orthogonal Designs. IEEE Trans. on Information Theory, 45(5):1456-1467, July 1999.

[8] J. William C. Jakes, editor. Microwave Mobile Communications. John Wiley and sons, New York, 1974.

[9] Shengli Zhou and Georgios B. Giannakis. Space-Time Coding With Maximum Diversity Gains Over Frequency-Selective Fading Channels. IEEE Signal Processing Letters, 8:269-272, October 2001.

[10] ETSI Normalization Committee. Channel Models for HIPERLAN/2 in different indoor scenarios. Norme ETSI, document 3ERI085B, European Telecommunications Standards Institute, Sophia-Antipolis, Valbonne, France, 1998.

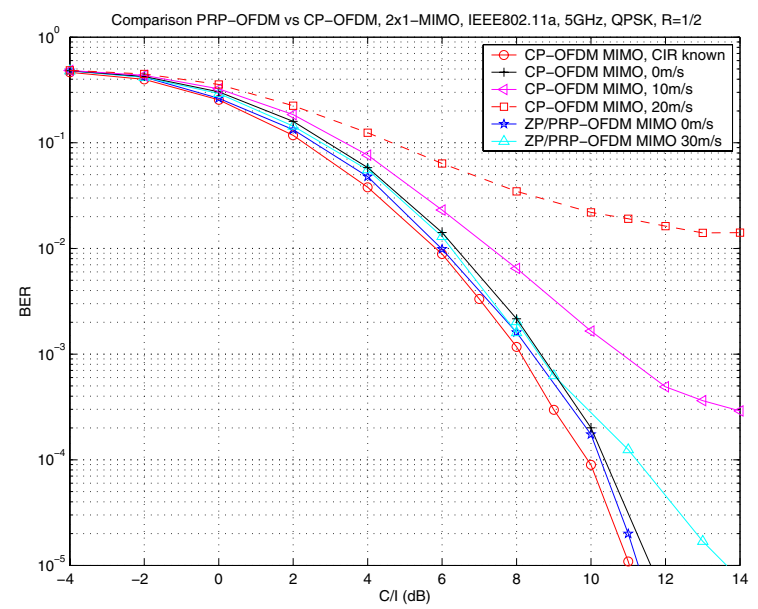

Fig. 2. Simulation results. 